$1-3-2014$

\title{
Expressing the Joint Moments of Drop Jumps and Sidestep Cutting in Different Reference Frames - Does it Matter?
}

\author{
Eirik Kristianslunda \\ Norwegian School of Sport Sciences \\ Tron Krosshauga \\ Norwegian School of Sport Sciences \\ Kam-Ming Mok \\ Norwegian School of Sport Sciences
}

BGIPdWMkfteqa additional works at: https://engagedscholarship.csuohio.edu/enme_facpub 'Iniversity of Michigan

Part of the Biomechanical Engineering Commons

Antprięes yae dejntBegertwork benefit you? Let us know!

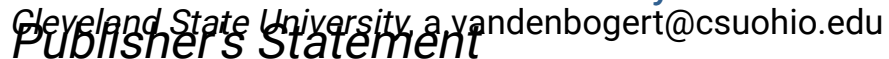

NOTICE: this is the author's version of a work that was accepted for publication in Journal of Biomechanics. Changes resulting from the publishing process, such as peer review, editing, corrections, structural formatting, and other quality control mechanisms may not be reflected in this document. Changes may have been made to this work since it was submitted for publication. A definitive version was subsequently published in Journal of Biomechanics, 47, 1, (01-03-2014); 10.1016/j.jbiomech.2013.09.016

\section{Original Citation}

Kristianslund, E., Krosshaug, T., Mok, K., 2014, "Expressing the Joint Moments of Drop Jumps and Sidestep Cutting in Different Reference Frames - does it Matter?" Journal of Biomechanics, 47(1) pp. 193-199. 


\title{
Expressing the joint moments of drop jumps and sidestep cutting in different reference frames - does it matter?
}

\author{
Eirik Kristianslund $^{\text {a, }}{ }^{\text {, }}$, Tron Krosshaug ${ }^{a}$, Kam-Ming Mok ${ }^{\mathrm{a}}$, Scott McLean ${ }^{\mathrm{b}}$, \\ Antonie J. van den Bogert ${ }^{\mathrm{c}}$ \\ aslo Sports Trauma Research Center, Norwegian School of Sport Sciences, PB 4014 Ulleval Stadion, 0806 Oslo, Norway \\ ${ }^{\mathrm{b}}$ School of Kinesiology. University of Michigan, US \\ 'Department of Mechanical Engineering, Cleveland State University, US
}

\section{Introduction}

Analyses of joint moments are at the core of mechanical analysis of human movement, and help us understand joint loading and muscle function. In standard three-dimensional (3D) motion analysis, net joint moments are calculated via an inverse dynamics approach (Bresler and Frankel, 1950). They can be expressed in different reference frames, e.g. the laboratory frame or the coordinate systems of the local segments adjacent to the joint (Andrews, 1984). The choice of reference frame depends primarily on the research questions and preferences, which may affect the interpretation of results (Winter and Ishac, 1994; Andrews, 1984; Schache and Baker, 2007). Joint moments expressed relative to a laboratory axis, for example, will represent this joint's contribution to movement in the plane perpendicular to that

\footnotetext{
* Corresponding author. Tel.: +47 40042 792; fax: +4723262307.

E-mail addresses: eirik.kristianslund@nih.no, eirik.kristianslund@gmail.com (E. Kristianslund).
}

axis. On the other hand, joint moments expressed relative to the local joint axes will represent the loading of the joint structures, and may be interpreted to correspond to muscle force production or ligament loading.

Lower extremity joint angles are usually calculated using the non-orthogonal axis system of the Joint Coordinate System (JCS), as recommended by the International Society of Biomechanics (Grood and Suntay, 1983; Wu and Cavanagh, 1995; Wu et al., 2002, 2005). Joint moments can be expressed relative to these axes to achieve correspondence between the joint angles and the joint moments, i.e. to ensure that a net flexion moment will result in a pure flexion (Andrews, 1984; Schache and Baker, 2007; Desroches et al., 2010). However, most commercial software systems have expressed joint moments in the orthogonal coordinate system of the distal segment of the joint and this has been commonly used in previous research (Dempsey et al., 2007; Chappell et al., 2002; Davis et al., 1991). The reasons for this choice are not clear, but it may be related to the use of local segment coordinate systems in the calculation of joint moments and the fact that a joint moment 
is a vector (unlike joint angle) and it is desirable to express it in an orthogonal axis system (Schache and Baker, 2007). In addition, the reflective markers over the tibia experience less soft-tissue artifacts than the femur markers, and Miranda et al. (2013) suggested that knee joint moments should be expressed in the tibia system to reduce the effects of soft-tissue artifacts.

Previous studies of standard gait have reported significant differences between joint moments expressed in different reference frames (Liu and Lockhart, 2006; Schache and Baker, 2007; Schache et al., 2007; Brandon and Deluzio, 2011). When comparing respective joint moments expressed in the global coordinate system, the local coordinate systems of the proximal and distal segment and the JCS, Schache et al. (2007) concluded that the frontal and transverse plane joint moments were more sensitive to a change of reference frame. This may affect conclusions from gait analysis, as Schache et al. (2008) found the effect of gait modification on knee adduction moments to be dependent on reference frame. However, Brandon and Deluzio (2011) reported results from gait analysis that were independent of reference frame. Subjects with osteoarthritis had reduced hip abduction moment and increased knee abduction moments during gait regardless if the joint moments were expressed in the global, distal or proximal frame, or the JCS.

Joint moments are important outcome variables in studies of drop jumps and sidestep cutting, tasks that involve a high range of motion and changes of direction (Besier et al., 2001; McLean et al., 2004; Hewett et al., 2005; Kristianslund and Krosshaug, 2013). These tasks are investigated particularly in studies of sport injury causation. An anterior cruciate ligament injury is one of the most serious sports injuries, based on its frequency and the serious consequences such as a long rehabilitation time and a high risk of early osteoarthritis (Renstrom et al., 2008). Knee abduction moments have been in focus as a risk factor for anterior cruciate ligament injury, and numerous studies on the knee abduction moment in drop jumps and sidestep cutting have been published (Besier et al., 2001; Hewett et al., 2005; McLean et al., 2005; Sigward and Powers, 2007; Carson and Ford, 2011; Benjaminse et al., 2011). With the direction changes and greater range of motion seen with drop jumps and sidestep cutting, the choice of reference frame may be even more important. However, the choice of reference frame is commonly not reported (Besier et al., 2001; Hewett et al., 2005; Sigward and Powers, 2007), and different methods are in use (Kristianslund and Krosshaug, 2013; McLean et al., 2005; Dempsey et al., 2007; Chappell et al., 2002). Standardization of joint moment reporting, similar to the ISB standard of joint angle reporting (Wu and Cavanagh, 1995), may improve the quality of reporting of results and facilitate comparison of studies, but this requires information on the differences between methods. Robinson and Vanrenterghem (2012) reported that the choice of knee axes may affect the kinetics of sidestep cutting, but the differences among different joint moment reference frames have only been investigated in gait.

Three reference frames are typically used in 3D motion analysis: the global laboratory frame (global), the local coordinate system of the distal segment (distal) and the JCS axes (Liu and Lockhart, 2006; Schache and Baker, 2007; Brandon and Deluzio, 2011). Two different methods can be used to express joint moments relative to the JCS axes: projection (JCSp) and decomposition (JCSd) (Desroches et al., 2010). The differences among methods stem from the difference in axis definitions and different methods to express joint moments relative to the axes. All methods but the JCSd use representations that equate projection of the joint moment vector to the relevant axes. The differences in orientation of the axes depend on their definitions and the orientation of body segments relative to the lab and to each other.

The aim of this investigation is examine the sensitivities of hip and knee joint moments for a drop jump and sidestep cutting task to four different calculation methods: global, distal, JCSp and JCSd. Respective calculation methods will be compared based on the difference between maximum values and the correlation of the ranking of trials based on maximum joint moments between methods.

\section{Methods}

Recordings from the baseline testing for a prospective risk factor study in elite Norwegian handball were used for this methodological study. The study was approved by the Regional Ethics Committee and all subjects signed informed consent forms.

\subsection{Testing and calculations}

Seventy female elite handball players (age $21.7 \pm 2.6$ years, weight $70.1+8.0 \mathrm{~kg}$, height $172+6 \mathrm{~cm}$ ) performed drop jumps and sidestep cuts in a motion analysis lab with eight $240 \mathrm{~Hz}$ infrared cameras (ProReflex, Qualisys, Gothenburg, Sweden) and two $960 \mathrm{~Hz}$ force platforms (AMTI, Watertown, Massachusetts, USA). Thirty-five reflective markers were attached as described previously (Kristianslund and Krosshaug, 2013). We performed a recording of the static anatomical position for each player prior to testing to define the anatomical coordinate systems.

Drop jumps were performed from a $30-\mathrm{cm}$ box. The athletes were instructed to drop off the box onto two force platforms and immediately perform a maximal jump. For sidestep cutting the players performed their usual sidestep cutting technique to pass a static human defender, cutting to the left (Fig. 1). They arrived at an angle of approximately $30^{\circ}$ to the long axis of the lab. Due to technique differences, the cutting angle ranged from $31^{\circ}$ to $110^{\circ}$ (mean \pm SD $67^{\circ} \pm 17^{\circ}$ ) and the approach speed from 2.3 to $4.2 \mathrm{~m} / \mathrm{s}(3.4 \pm 0.4 \mathrm{~m} / \mathrm{s})$. The defender adjusted her position to make sure the athlete hit the force platform using her self-selected sidestep cutting technique. Only trials where the athlete hit the force platform with all markers firmly attached to the skin and where the athlete displayed a matchlike effort, as assessed by an investigator and team mates, were used for analysis. The test procedures and calculations are described in detail previously (Kristianslund and Krosshaug, 2013).

Force and marker trajectories were processed with a smoothing spline with a $15 \mathrm{~Hz}$ cut-off frequency (Woltring, 1986; Kristianslund et al., 2012). Calculations were performed in custom Matlab scripts (MathWorks Inc, Natick, Massachusetts USA), with kinematics calculated according to the JCS convention (Grood and Suntay, 1983) and external joint moments calculated with iterative Newton-Euler inverse dynamics (Davis et al., 1991).

\subsection{Expression of joint moments}

Joint moments were expressed in four different reference frames (Fig. 2): the global laboratory frame ('global'), the local frame of the distal segment ('distal'), projected on to the JCS axes ('JCSp') and decomposed to JCS axes ('JCSd'). The expression of joint moments in different reference frames is defined in Eqs. (1)-(4)

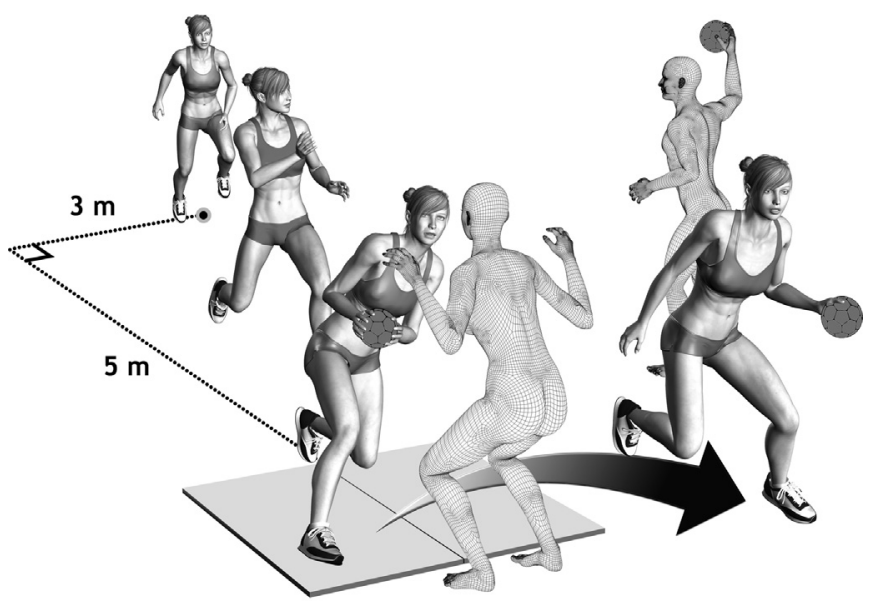

Fig. 1. Sidestep cutting situation. The players were instructed to try to fake the static defender into going to one side while cutting to the other. Prior to the cut, the player received the ball from a team mate in order to make the situation realistic Reproduced from Kristianslund et al. (2012). 


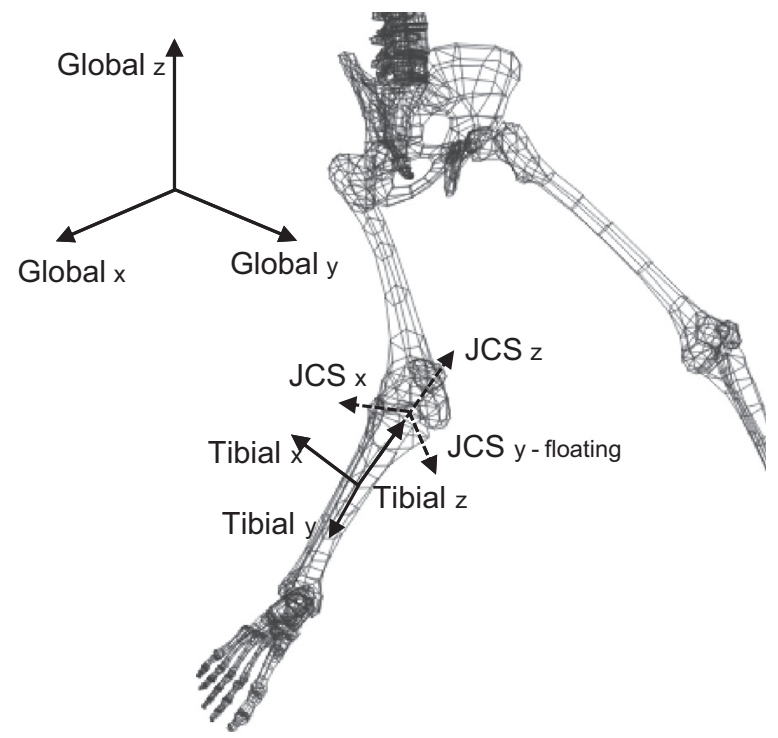

Fig. 2. Knee coordinate system used for the expression of joint moments. Global laboratory frame, tibia local frame and JCS axes. The tibial $z$ and the JCS $z$ are the same, JCS $x$ is the $x$-axis of the local femur frame and the JCS ${ }_{y}$ is the cross-product of the $\mathrm{JCS}_{x}$ and the $\mathrm{JCS}_{z}$.

The JCS flexion axis is defined as the medio-lateral axis of the proximal segment, the rotation axis as the longitudinal axis of the distal segment and the abduction axis as the cross-product of the flexion and rotation axes (Fig. 2). The rotation axes of the JCS and the distal segment are thus equal. However, flexion and abduction axes are different between the distal segment and the JCS. The flexion axis of the distal segment is different from the JCS flexion axis whenever there is abduction or rotation. The abduction axis differs between the distal segment and the JCS with rotation of the tibia relative to the femur, as the abduction axis of the JCS is mathematically independent of the rotation of the distal segment about its longitudinal axis.

As decomposition to a non-orthogonal system is different from projection to the axes of the non-orthogonal system, flexion and rotation moments in will be different from other representations even if the axes are the same when JCSd is applied. However, the abduction moment is the same with projection and decomposition, because the JCS abduction axis is the cross-product of the flexion and rotation axes (Desroches et al., 2010).

\subsection{Mathematical descriptions}

The net joint moment was first calculated in the global XYZ coordinate system, resulting in cartesian components $\mathbf{m}_{\text {global }}=\left(m_{X}, m_{Y}, m_{Z}\right)^{T}$, where $T$ indicates transpose. To express the joint moment in the local coordinate system of the distal or proximal segment, we use the rotation matrix which transforms from the global to the local coordinate system (Winter, 2009)

$\mathbf{R}_{\text {global } \rightarrow \text { local }}=\left(\mathbf{e}_{x}, \mathbf{e}_{y}, \mathbf{e}_{z}\right)^{T}$

where $\mathbf{e}_{x}, \mathbf{e}_{y}$ and $\mathbf{e}_{z}$ are unit vectors along the local coordinate axes, expressed in the global coordinate system. The joint moment expressed in local coordinates is then calculated as

$\mathbf{m}_{\text {local }} \equiv\left(\begin{array}{c}m_{x} \\ m_{y} \\ m_{z}\end{array}\right)=\mathbf{R}_{\text {global } \rightarrow \text { local }} \cdot \mathbf{m}_{\text {global }}$

During the kinematic analysis, we obtained unit vectors along the axes of the JCS, expressed in global cartesian coordinates. These are the flexion axis $\left(\mathbf{e}_{\text {flexion }}\right)$, the rotation axis ( $\mathbf{e}_{\text {rotation }}$ ), and the abduction axis $\left(\mathbf{e}_{\text {abduction }}\right)$ (Grood and Suntay, 1983).

JCSp joint moments were computed by taking dot products of the moment vector with unit vectors along the JCS axes

$\mathbf{m}_{\mathrm{JCSp}} \equiv\left(\begin{array}{c}m_{\mathrm{JCSp}, \text { flexion }} \\ m_{\mathrm{JCSp} \text {,abduction }} \\ m_{\mathrm{JCS} p \text {,rotation }}\end{array}\right)=\left(\begin{array}{c}\mathbf{e}_{\text {flexion }}^{T} \\ \mathbf{e}_{\text {abduction }}^{T} \\ \mathbf{e}_{\text {rotation }}^{T}\end{array}\right) \cdot \mathbf{m}_{\text {global }}$

Note that the matrix on the right hand side is not a rotation matrix because the rows are not orthogonal.
JCSd joint moments are defined as the three moments along the JCS axes that add up to the total moment vector

$m_{\mathrm{JCSd}, \text { flexion }} \cdot \mathbf{e}_{\text {flexion }}+m_{\mathrm{JCSd} \text {,abduction }} \cdot \mathbf{e}_{\mathrm{abduction}}+m_{\mathrm{JCS}, \text { rotation }} \cdot \mathbf{e}_{\text {rotation }}=\mathbf{m}_{\text {global }}$

This is a linear system of equations, with solution

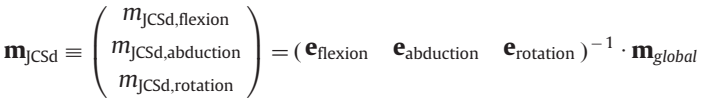

In a gimbal lock pose, when the flexion and rotation axes are parallel, the matrix inversion is not possible. Another special case occurs when the flexion and rotation axes are perpendicular. The matrix is then a rotation matrix, for which the inverse is equal to the transpose, and the JCSd joint moments will be identical to the JCSp joint moments.

Matlab code for the calculation of these joint moment expressions can be found online as Supplementary material.

\subsection{Statistical treatment}

Maximum hip and knee joint moments during the contact phase from three drop jumps and three sidestep cuts for each player were used for analysis. These were treated as originating from 210 individual drop jumps and 210 individual sidestep cuts, as we were interested in the differences between methods, not between subjects. All joint moments were normalized to body mass prior to statistical comparison. SPSS 18 (SPSS Inc., Chicago, IL, USA) was used for statistical analysis. The mean and standard deviation (SD) were used to describe the normalized joint moments.

To describe the magnitude of difference between methods, we calculated the root mean square (RMS) value of the difference between maximal values. This measure represents the typical difference between methods. To show the magnitude of the difference between methods relative to the typical difference between trials, we calculated the RMS in percent of the SD of maximum joint moment expressed in JCSp. The differences were expressed relative to the JCSp joint moments as this expression was part of all the chosen comparisons and is commonly used in previous research (McLean et al., 2005; Kristianslund et al., 2013).

In many applications of biomechanical analysis, the ranking of subjects based on the measures of interest is important. If the measures change in a somewhat systematic manner such that the ranking stays the same, the results of statistical analyses to describe group differences or injury risk may not change, as the grouping can be unaffected. The effect of expression method on the ranking of trials based on joint moment measures was described with Spearman's $\rho$.

We plotted the time series of joint moments from the most typical jump and sidestep cut (Fig. 3). We selected the trials with values closest to the average maximum joint moment for all hip and knee joint moments when expressed in JCSp and the most typical absolute difference for the most relevant comparisons. This was considered to be the difference between knee flexion, knee abduction, hip flexion, hip abduction moments expressed in JCSp and distal and the difference between knee flexion, knee internal rotation, hip flexion and hip internal rotation in JCSp and JCSd.

\section{Results}

The greatest differences between methods were seen for drop jumps hip internal rotation moments (range: $0.31-0.71 \mathrm{Nm} / \mathrm{kg}$ ) and sidestep cutting knee flexion $(2.87-3.39 \mathrm{Nm} / \mathrm{kg}$ ) and hip internal rotation $(0.87-2.36 \mathrm{Nm} / \mathrm{kg})$ and knee internal rotation $(0.10-0.40 \mathrm{Nm} / \mathrm{kg}$ ) moments (Table 1). The choice of reference frame to express joint moments clearly affected the ranking of subjects based on maximum joint moments (Table 2). The rank correlations were highest between conditions for flexion moments $(0.88-1.00)$ and sidestep cutting abduction moments (0.71-0.98). The rank correlations ranged from 0.64 to 0.73 for drop jump knee abduction moments and between -0.17 and 0.67 for hip and knee internal rotation moments. The RMS results demonstrate substantial differences in the magnitude of joint moments between methods (Table 3).

\section{Discussion}

We observed substantial differences among the expressions of joint moments calculated herein, both in ranking and magnitude of measures. Greatest differences in magnitude were seen for hip 

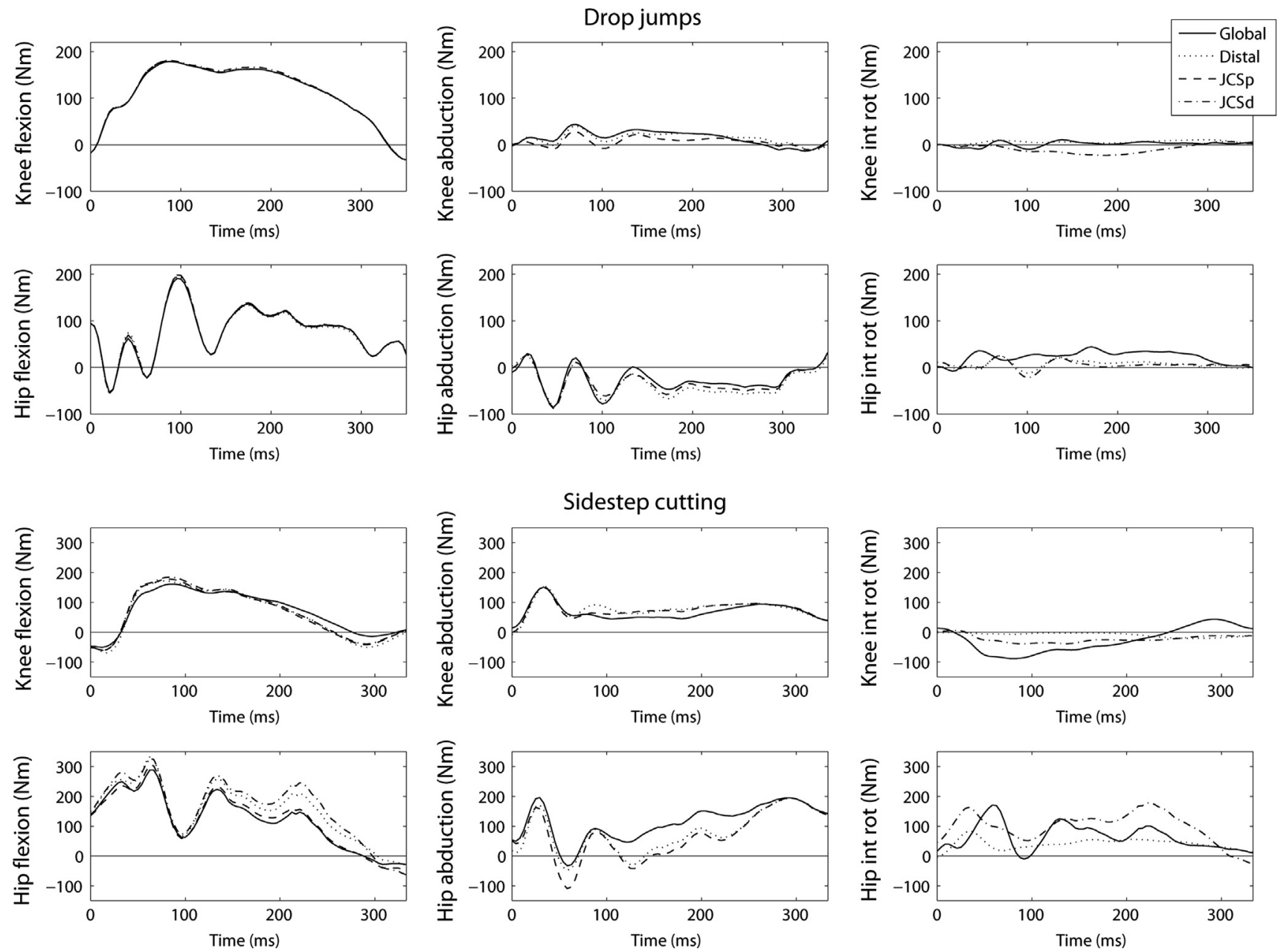

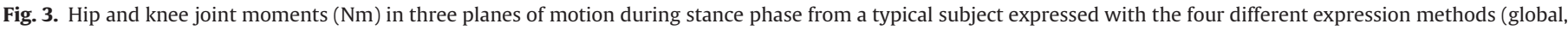
distal, JCSp and JCSd). Initial contact at $0 \mathrm{~ms}$. $Y$-axis labels relate to positive values.

Table 1

Mean (SD) maximum joint moment $(\mathrm{Nm} / \mathrm{kg})$ during contact phase. $N=210$ drop jumps and $N=210$ sidestep cuts.

\begin{tabular}{|c|c|c|c|c|}
\hline & Global & Distal & JCSp & JCSd \\
\hline \multicolumn{5}{|l|}{ Drop jumps } \\
\hline Knee flexion moment & $2.62(0.64)$ & $2.62(0.65)$ & $2.61(0.64)$ & $2.66(0.66)$ \\
\hline Knee abduction moment & $0.39(0.25)$ & $0.31(0.24)$ & $0.37(0.17)$ & $0.37(0.17)$ \\
\hline Knee internal rotation moment & $0.13(0.07)$ & $0.14(0.09)$ & $0.14(0.09)$ & $0.17(0.15)$ \\
\hline Hip flexion moment & $3.13(0.57)$ & $3.13(0.59)$ & $3.13(0.57)$ & $3.16(0.58)$ \\
\hline Hip abduction moment & $0.71(0.26)$ & $0.70(0.26)$ & $0.70(0.25)$ & $0.70(0.25)$ \\
\hline Hip internal rotation moment & $0.71(0.22)$ & $0.31(0.13)$ & $0.31(0.13)$ & $0.39(0.20)$ \\
\hline \multicolumn{5}{|l|}{ Sidestep cutting } \\
\hline Knee flexion moment & $2.87(0.56)$ & $3.35(0.62)$ & $3.26(0.61)$ & $3.39(0.63)$ \\
\hline Knee abduction moment & $1.72(0.79)$ & $1.94(0.75)$ & $1.94(0.72)$ & $1.94(0.72)$ \\
\hline Knee internal rotation moment & $0.40(0.14)$ & $0.12(0.16)$ & $0.12(0.16)$ & $0.10(0.18)$ \\
\hline Hip flexion moment & $3.90(1.07)$ & $4.57(1.19)$ & $4.15(1.11)$ & $4.59(1.11)$ \\
\hline Hip abduction moment & $3.00(0.83)$ & $2.78(0.83)$ & $2.64(0.84)$ & $2.64(0.84)$ \\
\hline Hip internal rotation moment & $2.36(0.72)$ & $0.87(0.31)$ & $0.87(0.31)$ & $1.87(0.62)$ \\
\hline
\end{tabular}

internal rotation moments in drop jumps and knee flexion and hip and knee internal rotation moments in sidestep cutting. The ranking was most consistent across conditions for knee and hip flexion moments in drop jumps and for knee and hip flexion and abduction moments in sidestep cutting, as described by the rank correlation between methods.

Flexion moments in drop jumps correlated well between methods, with small differences in magnitude. This was expected, as the movement was unidirectional and controlled, ensuring reasonable alignment of the flexion axes of the different reference frames. For sidestep cutting, only the global representation did not correlate well with the other methods. With a dynamic movement including directional change, like the sidestep cut, the local flexion axes cannot be expected to align with the "flexion axis" of the global laboratory system. However, the flexion axes of the distal segment and the JCS seem to be well aligned for both the hip and the knee, as the correlations were high and the magnitudes of differences were small. Still, there were quite high RMS differences 
Table 2

Rank correlation for maximum knee joint moments in expressed in different coordinate systems.

\begin{tabular}{|c|c|c|c|c|c|c|}
\hline & Global vs. Distal & Global vs. JCSp & Global vs. JCSd & Distal vs. JCSp & Distal vs. JCSd & JCSp vs. JCSd \\
\hline \multicolumn{7}{|l|}{ Drop jump } \\
\hline Knee flexion & 1.00 & 1.00 & 1.00 & 1.00 & 1.00 & \multirow[t]{2}{*}{1.00} \\
\hline Knee abduction & 0.64 & 0.73 & 0.73 & 0.72 & 0.72 & \\
\hline Knee internal rotation & 0.44 & 0.44 & 0.37 & & 0.67 & 0.67 \\
\hline Hip flexion & 0.99 & 0.99 & 0.99 & 0.99 & 0.99 & 1.00 \\
\hline Hip abduction & 0.90 & 0.93 & 0.93 & 0.95 & 0.95 & \\
\hline Hip internal rotation & 0.12 & 0.12 & -0.17 & & 0.66 & 0.66 \\
\hline \multicolumn{7}{|l|}{ Sidestep cutting } \\
\hline Knee flexion & 0.88 & 0.88 & 0.88 & 0.97 & 0.99 & \multirow[t]{2}{*}{0.97} \\
\hline Knee abduction & 0.91 & 0.91 & 0.91 & 0.98 & 0.98 & \\
\hline Knee internal rotation & -0.16 & -0.16 & -0.05 & & 0.39 & 0.39 \\
\hline Hip flexion & 0.95 & 0.95 & 0.90 & 0.97 & 0.96 & 0.94 \\
\hline Hip abduction & 0.75 & 0.71 & 0.71 & 0.95 & 0.95 & \\
\hline Hip internal rotation & 0.42 & 0.42 & 0.29 & & 0.61 & 0.61 \\
\hline
\end{tabular}

* mathematically equivalent expressions. $N=210$ drop jumps and $s s=210$ sidestep cuts.

Table 3

RMS for the difference in joint moments $(\mathrm{Nm} / \mathrm{kg})$ between methods in relevant comparison and these RMS in percent of SD of maximum joint moment expressed in JCSp. $N=210$ drop jumps and $N=210$ sidestep cuts.

\begin{tabular}{llllll}
\hline & \multicolumn{2}{l}{ Drop jumps } & & \multicolumn{2}{l}{ Sidestep cutting } \\
\cline { 2 - 3 } \cline { 6 - 7 } \cline { 5 - 6 } & RMS & RMS/SD & & RMS & RMS/SD \\
\hline Knee flexion JCSp/distal & 0.05 & $8 \%$ & & 0.17 & $27 \%$ \\
Knee flexion JCSp/JCSd & 0.08 & $12 \%$ & & 0.19 & $31 \%$ \\
Knee abduction JCSp/distal & 0.18 & $102 \%$ & & 0.16 & $22 \%$ \\
Knee int rot JCSp/JCSd & 0.12 & $140 \%$ & & 0.15 & $93 \%$ \\
Hip flexion JCSp/distal & 0.09 & $16 \%$ & & 0.52 & $47 \%$ \\
Hip flexion JCSp/JCSd & 0.05 & $9 \%$ & & 0.56 & $51 \%$ \\
Hip abduction JCSp/distal & 0.08 & $33 \%$ & & 0.26 & $31 \%$ \\
Hip int rot JCSp/JCSd & 0.18 & $134 \%$ & & 1.12 & $360 \%$ \\
\hline
\end{tabular}

for both hip and knee flexion moments in sidestep cutting when comparing JCSp to distal or JCSd. This indicates that even with small effects on ranking and mean maximum values, the results from the different methods are substantially different.

Abduction moments were affected by expression method, and the rank correlations between methods range from 0.64 to 0.95 . The ranking based on knee abduction moments was more consistent in sidestep cutting compared to drop jumps. The reasons for this are not clear, but it may be related to the fact that knee abduction moments occur earlier in the stance phase, with a lower knee flexion, in sidestep cutting compared to drop jumps (Kristianslund and Krosshaug, 2013). As can be seen from the plot of a typical trial, there were greater differences between methods in sidestep cutting later in the stance phase. Although the ranking was good between methods for knee abduction moments in sidestep cutting, there were substantial differences in magnitude, as shown by the RMS difference.

Internal rotation moments axes are very different between methods. The axis is the same for the JCS and distal methods, and the difference in axis orientation from the global axis is related to both flexion and abduction of the distal segment. There is no correlation between the global internal rotation moments and the distal and JCS internal rotation moments. When the local rotation axis is $90^{\circ}$ to the global axis, we expect no correlation at all. The local axis was not $90^{\circ}$ to the global during these movements, but the distal segment longitudinal axis was nevertheless far from being aligned with the global vertical axis. The difference between decomposing and projecting to the longitudinal axis affected both magnitude and rank correlation of trials.
This study demonstrated differences in joint moment measures among various reference frames, as have previously been reported in standard gait (Schache et al., 2007; Schache and Baker, 2007; Liu and Lockhart, 2006). We have also shown how this can affect the ranking of athletes. Consequently, the choice of reference frame has the potential to affect conclusions of biomechanical studies analyzing knee and hip kinetics of drop jumps and sidestep cutting. There is an abundance of such studies to elucidate anterior cruciate ligament injury causation, but the lack of consistency in joint moment calculation and interpretation makes it difficult to compare studies and develop strong assertions regarding injury causation. The RMS difference between e.g. distal and JCSp knee abduction moments are similar to mean sex differences seen previously (McLean et al., 2005) and approaches the magnitude of the effect of fatigue on knee abduction moments (McLean et al., 2007).

In joint injury research, we are primarily interested in the loading of the joint, not its contribution to movement in a certain direction. Global joint moments may represent the latter, while local joint-specific expressions of joint moments are necessary to interpret joint moments as representing muscle force or ligament loading. The tibia reference frame is less affected by soft tissue artifacts (Miranda et al., 2013) and has the attractive properties of being an orthogonal coordinate system. On the other hand, expressing knee joint moments in this reference frame may not provide joint moment measures that correspond to our understanding of what a joint moment is. If we express knee flexion moments in the tibia system, they will not correspond to the extension moment generated by the quadriceps muscles if the knee is internally or externally rotated.

Expressing joint moments in the JCS facilitates interpretation of results and is a natural choice in multi-planar motion (Schache and Baker, 2007). This ensures a correspondence between joint kinematics and kinetics which may not be seen if joint moments are expressed in other reference frames. As the rank correlations between the JCS expressions and the other expression methods were moderate or poor for some of the joint moments, misguided conclusions may result from expressing in frames other than the kinematic joint axes. Furthermore, with only moderate correlation of internal rotation moments projected or decomposed to JCS axes, it is also important to consider how joint moments are expressed in the JCS.

The interpretations of the projection and decomposition of joint moments to JCS axes are different. Mechanically, the JCS can be considered hinge joints in series (Grood and Suntay, 1983). The net joint moment projected to one of the joint axis will describe the torque that is needed to resist the load on that axis (Desroches 
et al., 2010). If the net joint moment is decomposed to these nonorthogonal axes, joint moment vectors will sum up to the net joint moment, but the interpretation of the joint moment about an axis will be unclear. When interpreting local joint moments in light of muscle and ligament function, we want to know the torques that the muscles and ligaments need to resist about our chosen axis, and this will be described by projecting the net joint moment onto the axis.

This study is based on one test setup and test protocol only, and results may be different in other labs or with other test procedures. The kinematics and kinetics from 3D motion analysis depend on a number of factors, such as skin movement, data filtering, joint center determination and segment coordinate system calculations (Chiari et al., 2005; Leardini et al., 2005; Della Croce et al., 2005; Kristianslund et al., 2012). However, in this study the only difference between the compared methods was how we expressed joint moments, and we have described the differences between expressions of joint moments in a typical testing situation.

\section{Conclusion}

The choice of reference frame to express hip and knee joint moments significantly affects the calculated joint moments during dynamic high-impact landing movements. This is especially true for abduction and internal rotation moments, where there may be only a poor or moderate correlation of the ranking of subjects between different methods. The choice of method may influence the conclusions of biomechanical studies examining such movements, and joint moments should be interpreted in light of the methods used to obtain them. Consequently, the method used to express joint moments should be reported in mechanical analysis of human movement. Furthermore, a standardization of joint moment reporting could facilitate comparison of studies and improve the quality of data generation and interpretation. As projection to the JCS is the only method where joint moments correspond to muscle and ligament loading, it is a natural choice for a standard of joint moment reporting.

\section{Conflict of interest statement}

No author has any financial and personal relationships with other people or organisations that could inappropriately influence their work.

\section{Acknowledgments}

The Oslo Sports Trauma Research Center has been established at the Norwegian School of Sport Sciences through generous grants from the Royal Norwegian Ministry of Culture, the SouthEastern Norway Regional Health Authority, the International Olympic Committee, the Norwegian Olympic Committee \& Confederation of Sport, and Norsk Tipping AS.

\section{Appendix A. Supplementary material}

Supplementary data associated with this article can be found in the online version at http://dx.doi.org/10.1016/j.jbiomech.2013.09.016.

\section{References}

Andrews, J.G., 1984. On the specification of joint configurations and motions Journal of Biomechanics 17, 155-158.
Benjaminse, A., Gokeler, A., Fleisig, G.S., Sell, T.C., Otten, B., 2011. What is the true evidence for gender-related differences during plant and cut maneuvers? A systematic review. Knee Surg Sports Traumatology Arthroscopy 19, 42-54.

Besier, T.F., Lloyd, D.G., Cochrane, J.L., Ackland, T.R., 2001. External loading of the knee joint during running and cutting maneuvers. Medicine and Science in Sports and Exercise 33, 1168-1175.

Brandon, S.C., Deluzio, K.J., 2011. Robust features of knee osteoarthritis in joint moments are independent of reference frame selection. Clinical Biomechanics 26, 65-70.

Bresler, E., Frankel, J.P., 1950. The forces and moments in the leg during level walking. Journal of Applied Mechanics, 27-36.

Carson, D.W., Ford, K.R., 2011. Sex differences in knee abduction during landing. Sports Health 3, 373-382.

Chappell, J.D., Yu, B., Kirkendall, D.T., Garrett, W.E., 2002. A comparison of knee kinetics between male and female recreational athletes in stop-jump tasks. American Journal of Sports Medicine 30, 261-267.

Chiari, L., Della, C.U., Leardini, A., Cappozzo, A., 2005. Human movement analysis using stereophotogrammetry. Part 2: instrumental errors. Gait Posture 21, 197-211.

Davis III, R.B., Ounpuu, S., Tyburski, D., Gage, J.R, 1991. A gait analysis data collection and reduction technique. Human Movement Science 10, 575-578.

Della Croce, U., Leardini, A, Chiari, L., Cappozzo, A, 2005, Human movement analysis using stereophotogrammetry. Part 4: assessment of anatomical landmark misplacement and its effects on joint kinematics. Gait Posture 21, $226-237$.

Dempsey, A.R., Lloyd, D.G., Elliott, B.C., Steele, J.R., Munro, B.J., Russo, K.A., 2007. The effect of technique change on knee loads during sidestep cutting. Medicine and Science in Sports and Exercise's 39, 1765-1773.

Desroches, G., Cheze, L., Dumas, R., 2010. Expression of joint moment in the joint coordinate system. Journal of Biomechanical Engineering 132, 114503.

Grood, E.S., Suntay, W.J., 1983. A joint coordinate system for the clinical description of three-dimensional motions: application to the knee. Journal of Biomechanical Engineering 105, 136-144.

Hewett, T.E., Myer, G.D., Ford, K.R., Heidt Jr., R.S., Colosimo, A.J., McLean, S.G., van den Bogert, A.J., Paterno, M.V., Succop, P., 2005. Biomechanical measures of neuromuscular control and valgus loading of the knee predict anterior cruciate ligament injury risk in female athletes: a prospective study. American Journal of Sports Medicine 33, 492-501.

Kristianslund, E., Faul, O., Bahr, R., Myklebust, G., Krosshaug, T., 2013. Sidestep cutting technique and knee abduction loading: implications for ACL prevention exercises. British Journal of Sports Medicine. (Epub ahead of print).

Kristianslund, E., Krosshaug, T., 2013. Comparison of drop jumps and sport-specific sidestep cutting: implications for anterior cruciate ligament injury risk screening. American Journal of Sports Medicine 41, 684-688.

Kristianslund, E., Krosshaug, T., van den Bogert, A.J., 2012. Effect of low pass filtering on joint moments from inverse dynamics: implications for injury prevention. Journal of Biomechanics 45, 666-671.

Leardini, A. Chiari, L., Della, C.U., Cappozzo, A. 2005. Human movement analysis using stereophotogrammetry. Part 3. Soft tissue artifact assessment and compensation. Gait Posture 21, 212-225.

Liu, J., Lockhart, T.E., 2006. Comparison of 3D joint moments using local and global inverse dynamics approaches among three different age groups. Gait Posture 23, 480-485.

McLean, S.G., Felin, R.E., Suedekum, N., Calabrese, G., Passerallo, A., Joy, S., 2007. Impact of fatigue on gender-based high-risk landing strategies. Medicine and Science in Sports and Exercise 39, 502-514

McLean, S.G., Huang, X., van den Bogert, A.J., 2005. Association between lower extremity posture at contact and peak knee valgus moment during sidestepping: implications for ACL injury. Clinical Biomechanics 20, 863-870.

McLean, S.G., Lipfert, S.W., van den Bogert, A.J., 2004. Effect of gender and defensive opponent on the biomechanics of sidestep cutting. Medicine and Science in Sports and Exercise 36, 1008-1016.

Miranda, D.L., Rainbow, M.J., Crisco, J.J., Fleming, B.C., 2013. Kinematic differences between optical motion capture and biplanar videoradiography during a jumpcut maneuver. Journal of Biomechanics 46, 567-573.

Renstrom, P., Ljungqvist, A., Arendt, E., Beynnon, B., Fukubayashi, T., Garrett, W. Georgoulis, T., Hewett, T.E., Johnson, R., Krosshaug, T., Mandelbaum, B., Micheli, L., Myklebust, G., Roos, E., Roos, H., Schamasch, P., Shultz, S., Werner, S., Wojtys, E., Engebretsen, L., 2008. Non-contact ACL injuries in female athletes: an international olympic committee current concepts statement. British Journal of Sports Medicine 42, 394-412.

Robinson, M.A., Vanrenterghem, J., 2012. An evaluation of anatomical and functional knee axis definition in the context of side-cutting. Journal of Biomechanics 45, 1941-1946.

Schache, A.G., Baker, R., 2007. On the expression of joint moments during gait. Gait Posture 25, 440-452.

Schache, A.G., Baker, R., Vaughan, C.L., 2007. Differences in lower limb transverse plane joint moments during gait when expressed in two alternative reference frames. Journal of Biomechanics 40, 9-19.

Schache, A.G., Fregly, B.J., Crossley, K.M., Hinman, R.S., Pandy, M.G., 2008. The effect of gait modification on the external knee adduction moment is reference frame dependent. Clinical Biomechanics 23, 601-608.

Sigward, S.M., Powers, C.M., 2007. Loading characteristics of females exhibiting excessive valgus moments during cutting. Clinical Biomechanics 22, 827-833.

Winter, D.A., 2009. Biomechanics and Motor Control of Human Movement. Wiley. 
Winter, D.A., Ishac, M.G., 1994. XIVth International society of biomechanics congress: interpretation of 3D moments-of-force during gait. Journal of Biomechanics 27, 818 .

Woltring, H.J., 1986. A Fortran package for generalized, cross-validatory spline smoothing and differentiation. Advances in Engineering Software 8, 104-113.

Wu, G., Cavanagh, P.R., 1995. ISB recommendations for standardization in the reporting of kinematic data. Journal of Biomechanics 28, 1257-1261.

Wu, G., Siegler, S., Allard, P., Kirtley, C., Leardini, A., Rosenbaum, D., Whittle, M., D’Lima, D.D., Cristofolini, L., Witte, H., Schmid, O., Stokes, I., 2002. ISB recommendation on definitions of joint coordinate system of various joints for the reporting of human joint motion-part I: ankle, hip, and spine. International society of biomechanics. Journal of Biomechanics 35, 543-548.

Wu, G., van der Helm, F.C., Veeger, H.E., Makhsous, M., Van, R.P., Anglin, C., Nagels, J., Karduna, A.R., McQuade, K., Wang, X., Werner, F.W., Buchholz, B., 2005. ISB recommendation on definitions of joint coordinate systems of various joints for the reporting of human joint motion-Part II: shoulder, elbow, wrist and hand. Journal of Biomechanics 38, 981-992. 